\title{
Aldosteronantagonisten bei Herzinsuffizienz selten verordnet
}

\author{
Weniger als ein Drittel der geeig- \\ neten Herzinsuffizienzpatienten, \\ die stationär behandelt werden, \\ bekommen in den USA Aldosteron- \\ antagonisten.
}

- Im Rahmen eines Qualitätssicherungsprogramms führte man eine Beobachtungsstudie an 43625 Patienten mit Herzinsuffizienz durch, die in 241 amerikanischen Krankenhäusern behandelt worden waren und nach Hause entlassen werden sollten. Unter Beachtung der einschlägigen Kontraindikationen fanden sich 12565 Patienten, bei denen eine Therapie mit Aldosteronantagonisten indiziert gewesen wäre. Tatsächlich erhielten nur $4087 \mathrm{~Pa}-$ tienten $(32,5 \%)$ bei Entlassung diese Therapie. Im Verlauf der Studiendauer von Januar 2005 bis Dezember 2007 nahm der Anteil der so behandelten Patienten immerhin von 28 auf $34 \%$ zu.

Der Einsatz von Aldosteronantagonisten war mit jüngerem Lebensalter, afroamerikanischer ethnischer Herkunft, einem niedrigeren systolischen Blutdruck, dem Zustand nach Implantation eines ICD, einer Depression, einem schädlichen Alkoholgebrauch und einer Schrittmacherimplantation assoziiert. Auffallend war eine weite Varianz der Anwendung von Aldosteronantagonisten in verschiedenen Krankenhäusern, die von den Extremwerten $0 \%$ bis über $90 \%$ reichte. Offensichtlich gibt es Krankenhäuser, bei denen Aldosteronantagonisten bei der Herzinsuffizienz nahezu tabu sind.

Der Umstand, dass dagegen kaum Patienten mit Aldosteronantagonisten behandelt wurden, bei denen die An- wendung kontraindiziert oder zumindest fraglich indiziert gewesen wäre, kann als Hinweis gelten, dass man unerwünschte Arzneimittelwirkungen, insbesondere bei Patienten mit Neigung zur Hyperkaliämie oder Niereninsuffizienz, befürchtet. An dieser Größe änderte sich im Lauf der dreijährigen Studienperiode auch nichts.

KOMMENTAR: Insbesondere in Deutschland ist es guter Brauch, auf das nicht leitlinienkonforme Verhalten von Ärzten anklagend hinzuweisen. Die Studie zeigt, dass diese Probleme durchaus auch in anderen Ländern existieren.

H. S. FÜEßL =
N. M. Albert et al.
Use of aldosterone antagonists in heart failure. JAMA 302 (2009) 15, 1658-1665

\section{Diabetes mellitus Typ 1 auf dem Vormarsch}

Wenn der Trend der letzten 15 Jahre anhält, so ist bis zum Jahr 2020 mit einem Anstieg der Prävalenz des Typ-1-Diabetes bei Kindern unter 15 Jahren um 70\% zu rechnen.

- Epidemiologen des EURODIAB-Registers haben in den Jahren 1989-2003 in 17 Ländern 29311 neue Fälle von Typ-1-Diabetes bei Kindern unter 15 Jahren registriert. In 18 von 20 Populationsregistern beobachtete man einen signifikanten jährlichen Zuwachs der Inzidenz, der sich zwischen 0,6 und 9,3\% bewegte. Insgesamt nahmen pro Jahr die Fälle von Typ-1-Diabetes um 9,3\% zu.

Die Verteilung in den Altersgruppen zeigte, dass sich die Entwicklung gerade in den letzten Jahren beschleunigt hat. Bei den Zehn- bis Vierzehnjährigen betrug die Zunahme 2,9\%, bei den Fünf- bis Neunjährigen 4,3\%, bei den Null- bis Vierjährigen sogar 5,4\%.

Im Jahr 2005 dürften etwa 15000 neue Fälle von Typ-1-Diabetes aufgetreten sein, davon $24 \%$ bei den
Null- bis Vierjährigen, 35\% bei Fünf- bis Neunjährigen und $41 \%$ bei den Zehn- bis Vierzehnjährigen. Für das Jahr 2020 wird eine Zahl von 24400 neuen Fällen prognostiziert, mit einer Verdoppelung der Diabetesfälle bei den Kindern unter fünf Jahren und einer nahezu gleichmäßigen Verteilung über die drei genannten Altersgruppen.

KOMMENTAR: Die Zunahme der Fälle von Typ-1-Diabetes hat Konsequenzen für die Vorhaltung von stationären Behandlungseinrichtungen. Es ist damit zu rechnen, dass in den nächsten zehn Jahren mehr Kinder mit diabetischer Ketoazidose erkranken werden, die einer intensivmedizinischen Behandlung bedürfen.

H. S. FüEßL =

\section{- C. C. Patterson et al.}

(Korr.: Christopher C. Patterson, MD Centre for Public Health, Queen's University Belfast, Mulhouse Building, Grosenor Road, Belfast BT 12 6BJ, UK, e-mail: c.patterson@qub.ac.uk): Incidence trends for childhood type 1 diabetes in Europe during 1989-2003 and predicted new cases 2005-20: a multicentre prospective registration study. Lancet 373 (2009) 9680, 2027-2033 